\title{
Multi-Resonant based Output Voltage Control of Autonomous Distributed Generators
}

\author{
Abdelhakim Saim ${ }^{1}$, Rabah Mellah ${ }^{2}$, and Azeddine Houari ${ }^{3}$ \\ ${ }^{1}$ Faculty of Electrical Engineering, University of Science and Technology Houari Boumedien (USTHB), BP n³2, El Alia - Bab Ezzouar- \\ 16111, Algiers, Algeria \\ ${ }^{2}$ L2CSP Laboratory, Faculty of Electrical and Computing Engineering, University Mouloud Mammeri of Tizi-Ouzou, 15000, Tizi- \\ Ouzou, Algeria \\ ${ }^{3}$ IREENA Laboratory, University of Nantes, 44602, Saint-Nazaire, France
}

\begin{abstract}
The distributed or decentralized generation electricity constitutes the central stone of various recent energy models, such as the intelligent electrical supply networks. The quality of energy in this type of structure depends primarily on the strategy of control adopted, in order to guarantee proper operation according to the international standards. The strategy of control proposed in this paper uses two control loops. An internal control loop aims at deadening the phenomena of resonance while ensuring the required control dynamic for fast disturbance rejection. As for the external control loop, a multi-resonant composed of a stabilizing state feedback that ensure an asymptotic tracking of the voltage reference with a weak rate of harmonic distortion. Finally, experimental results are presented to show the performances and feasibility of the proposed control strategy.
\end{abstract}

\section{Introduction}

The autonomous systems of distributed generation appropriate to electricity require an adequate and permanent control of the tension of the bus AC in order to ensure a quasi sinusoidal tension with a weak rate of harmonic distortion (THD), and this, independently of the dynamics of the fed electric charges. Moreover, the autonomous electrical supply networks with low tension (BT) are regarded as fragile structures because of the difficulties related to the maintenance of a bus tension with amplitude and frequency constants. These difficulties are exacerbated by the proliferation of the electric charges with strong not linearity, as by the intermittency which characterizes the majority of the sources of renewable energies. The face values of the amplitude and the frequency of tension are imposed by the requirements related to the electric charges (domestic or industrial) and those of the common electrical supply network. However, the variations can be introduced by the primary control of flows of power in the case of several inter-connected distributed generators.

Several strategies of controllers are proposed in the literature with a common aim to maintain the output voltage of the inverters on satisfactory levels [1, 2]. Among the principal strategies of control which exist in the literature, the resonant controllers (PR) can bring a solution to the problems related to the follow-up of periodic references. Indeed, resonant control provides profits very high to a precise frequency what makes it possible to eliminate, in closed loop, any static error at this frequency. Nevertheless, the selective character of this type of control with regard to the frequency of resonance conduit with the drastic deterioration of the performances of control even during light variations of the frequency of the bus AC [3-4]. Thus, application of the resonant controllers in the control of the autonomous systems of distributed generation of electricity must face the recurring variations of frequency in the micro grid network [6]. Moreover, research in progress, is focused on the design of a multi resonant controller who is more robust with regard to the fluctuation of the frequency all while maintaining the effectiveness of the multi resonant controllers in the follow-up and the rejection of periodic signals.

In this context, the work developed in this paper aims to bring solutions allowing the improvement of the quality of the energy of an autonomous micro grid comprising several distributed units of generation.

Control level relates to the regulation of the output voltage of the generator via the concept of resonant control. Indeed, resonant control positions from its performances among the techniques of control the most adapted for the follow-up and/or the rejection of periodic references, but suffers nevertheless certain gaps dependent primarily on its instability and its sensitivity to the variation of the point of operation (frequency) [7]. This can affect these performances harshly and limit its application in the systems of generation distributed considering the wave of the tension in these systems is the subject constantly of recurring variations in frequency and amplitude [8]. In this direction, a solution is proposed in order to benefit from the concept of resonant control without limiting its application. This solution consists in using a multi-resonant controller on stabilizing feedback 
states [9], whose profits of control are obtained from the minimization of a quadratic criterion.

Moreover, a bench of experimental test is built to validate the performances of the strategy of control developed using many experimental results before finishing with some conclusion.

\section{Description of studied system}

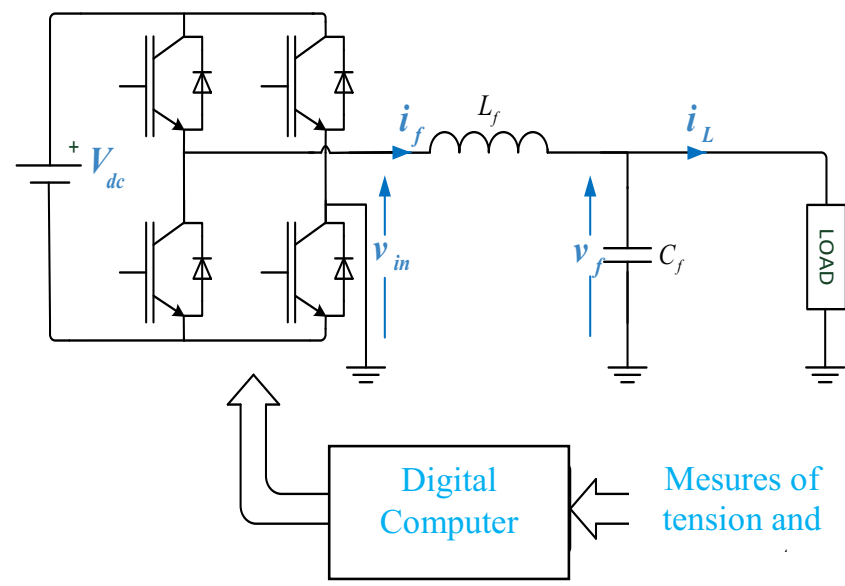

Fig. 1. Power circuit of an autonomous generator distributed.
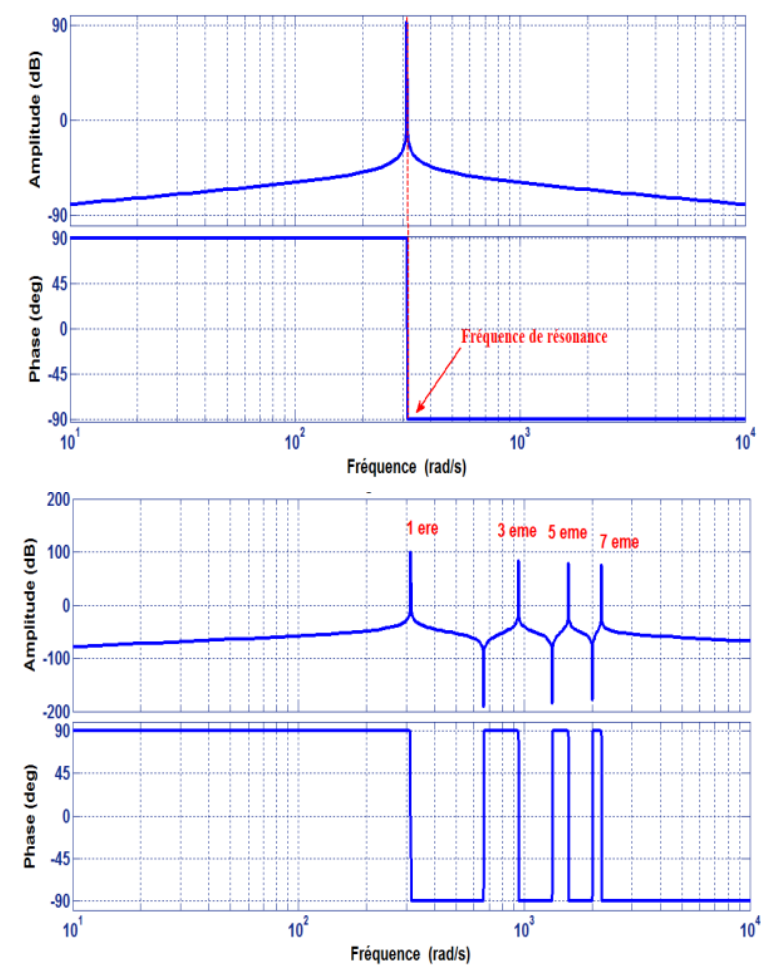

Fig. 2. Diagram of Bode, an ideal resonant controller (first), and of a multi-resonant controller (second).

The studied system consists of an autonomous unit of distributed generation of electricity composed mainly by an inverter source of tension whose control must guarantee at exit a wave of tension with high quality independently of the nature fed loads. This structure is illustrated by Fig. 1. The latter includes an inverter single-phase current controlled in modulation of width of impulse, MLI, a filter LC of exit whose role is to reduce the high frequency harmonic contents due to cutting as well as a bus AC represented by an unknown load [1011].

The circuit proposed does not consider the use of a transformer considering the use of this last is preferred in three-phase systems, because not only supporting the capacities of insulation of the system and providing a means of transformation but also to provide a way appropriate to the homopolar component of the currents. Thus, the distributed generator proposed is designed to deliver directly on the bus of the loads. The operation of the converter of power is controlled by a strategy of adequate control implemented in a digital computer whose objective is to generate the optimal states of commutations in order to ensure at exit the desired signals.

\section{Resonant controllers}

The resonant corrector takes then, Like illustrated by Fig. 2 , a theoretically infinite value at the frequency determined by the indicated poles. The principle of the resonant corrector rises from the theory of the internal models which stipulates that to facilitate the follow-up of the references and the rejection of the disturbances, it is necessary to integrate the models of these signals in the loop of control [12-16]. The transfer function of this corrector is given by:

$$
C_{R}(s)=\frac{k_{R} s}{s^{2}+\omega_{R}^{2}}
$$

The transfer function of the ideal resonant corrector (3.1) has purely imaginary poles. With the result that it is able to correct any statistical error of the same frequency than its frequency from resonance, than it is in the follow-up or the rejection of alternating signals. Where $\omega_{R}$ the angular frequency of resonance of corrector and $k_{R}$ the gain of control.

An assembly of several resonant controllers of various frequencies is possible. What increases the attractively of this type of control in the systems of distributed generation of electricity. Consequently, the control of the fundamental component of the output signals must be accompanied by control by the harmonic components. From where the transfer function of the corrector:

$$
C_{\text {Multi-R }}(s)=\sum_{h=1, o d d}^{n} \frac{k_{R, h} s}{s^{2}+\left(h \times \omega_{R}\right)^{2}}
$$

\section{Multi resonant control with stabilizing feedback states}

The strategy of control proposed is contingent, as Fig. 3 shows it, on a structure in cascade where the control of the tension $v_{f}$ of output will be done by ensuring the control of the current $i_{f}$. Thus, each loop of control will 
be seen charged to provide the adequate reference to the loop which follows.

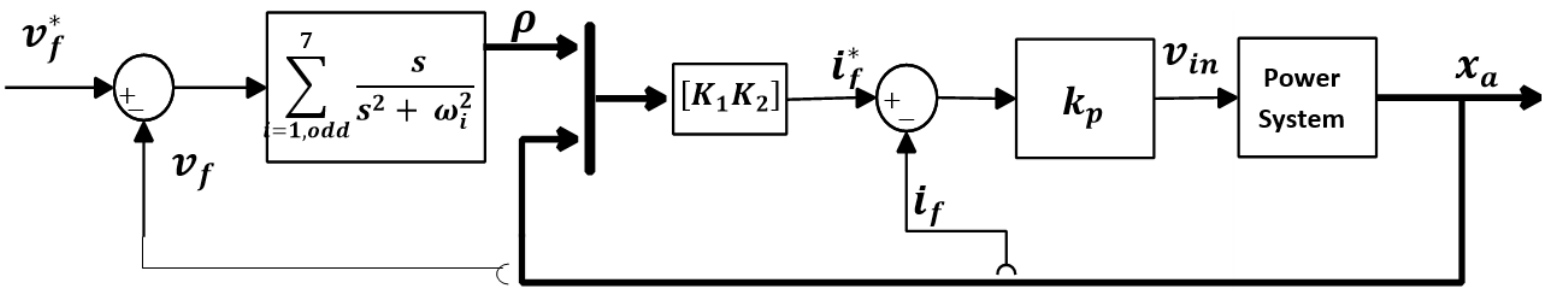

Fig. 3. Multi-resonant Controller with stabilizing return of states.

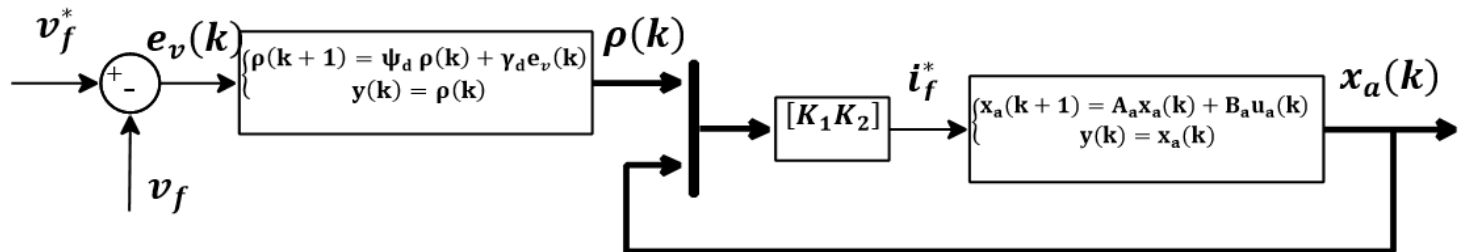

Fig. 4. State representation of the global system.

The internal loop of control aims to deadening the phenomena of resonance while ensuring dynamics necessary for a fast rejection of the disturbances. This loop uses the following law of control:

$$
v_{i n}(k+1)=k_{p}\left(i_{f}^{*}(k)-i_{f}(k)\right)
$$

Where $k_{p}$ is the proportional gain of the controllor and $i_{f}^{*}$ is the reference of the output current.

The external loop of control considers for it the use of a multi resonant controller with a complete stabilizing state feedback. It aims to ensure an asymptotic follow-up of the reference voltage standard with a reduced rate of harmonic distortion.

This loop employs the following law of control:

$$
i_{f}^{*}=\boldsymbol{K}_{\mathbf{1}} \boldsymbol{x}_{\boldsymbol{a}}+\boldsymbol{K}_{\mathbf{2}}\left[\sum_{i=1, o d d}^{7} \frac{S}{S^{2}+\omega_{i}^{2}}\right] e_{v}
$$

The error of follow-up is defined by: $e_{v}=v_{f}^{*}-v_{f}$. The centre frequency of the resonant controllers is $\omega_{i}=$ $2 \times \pi \times f_{0} \times i$, where $f_{0}$ represent the fundamental frequency of the system, and the argument $i$ refer to fundamental or the order of the harmonic concerned. The choice of the vectors of gains $\boldsymbol{K}_{1}$ and $\boldsymbol{K}_{\mathbf{2}}$ is done in an optimal way, after the resolution of a quadratic criterion. $\boldsymbol{x}_{\boldsymbol{a}}$ represent the state vector of the increased system.

\subsection{State representation of the increased system}

The state representation of the increased system is given by:

$$
\left\{\begin{array}{c}
x_{a}(k+1)=A_{a} x_{a}(k)+B_{a} u_{a}(k)+E_{a} d(k) \\
y(k)=C_{a} x_{a}(k)
\end{array}\right.
$$

The state vector $x_{a}(k)=\left[\begin{array}{lll}v_{f}(k) & i_{f}(k) & v_{i n}(k)\end{array}\right]^{T}$ The control signal $u_{a}(k)=i_{f}^{*}(k)$

$$
\begin{gathered}
A_{a}=\left[\begin{array}{ccc}
a_{11} & a_{12} & b_{1} \\
a_{21} & a_{22} & b_{2} \\
0 & -k_{p} & 0
\end{array}\right], B_{a}=\left[\begin{array}{c}
0 \\
0 \\
k_{p}
\end{array}\right] E_{a}=\left[\begin{array}{c}
e_{1} \\
e_{2} \\
0
\end{array}\right] \text { and } \\
C_{a}=\left[\begin{array}{ll}
C & 0
\end{array}\right]
\end{gathered}
$$

\section{2 State representation of multi resonant controller}

The state form of the multi resonant controller used is given as follows:

$$
\frac{d \rho}{d t}=\psi \rho+\gamma e_{v}
$$

Where

And

$$
\begin{gathered}
\rho=\left[\begin{array}{lll}
\rho_{1} & \rho_{3} \rho_{5} & \rho_{7}
\end{array}\right]^{T} \\
\psi=\operatorname{diag}\left[\begin{array}{lll}
\psi_{1} & \psi_{3} \psi_{5} & \psi_{7}
\end{array}\right] \\
\gamma=\left[\begin{array}{lll}
\gamma_{1} & \gamma_{3} \gamma_{5} & \gamma_{7}
\end{array}\right]^{T}
\end{gathered}
$$

$$
\psi_{i}=\left[\begin{array}{cc}
0 & 1 \\
-\omega_{i} & 0
\end{array}\right], \gamma_{i}=\left[\begin{array}{l}
0 \\
1
\end{array}\right], \text { for } i=1,3,5,7
$$

The discrete state representation of the multi resonant controller is given by:

$$
\rho(k+1)=\psi_{d} \rho(k)+\gamma_{d} e_{v}(k)
$$

Where

$$
\psi_{d}=e^{\psi T_{s}} \mathrm{et} \gamma_{d}=\int_{0}^{T_{s}} e^{\psi\left(T_{s}-\tau\right)} \gamma d \tau
$$

\subsection{Total representation of the system.}

(5) The optimal strategy of control employed corresponds to a structure of linear quadratic regulation (LQR) of which the resolution requires, in first, the total state representation of the system (Fig. 4) in order to carry out 
the placement of the eigenvalues of the state matrix via a single choice of the gain vectors $\boldsymbol{K}_{\mathbf{1}}$ and $\boldsymbol{K}_{\mathbf{2}}$

Thus, the total state representation of the system can be written as follows:

$$
\hat{x}(k+1)=\hat{A} \hat{x}(k)+\hat{B} u_{a}(k)+\widehat{E_{1}} d(k)+\widehat{E_{2}} v_{f}^{*}(k)
$$

Where $u_{a}(k)=i_{f}^{*}(k)$ represent the control signal, the state vector is define by $\hat{x}(k)=\left[x_{a}(k) \rho(k)\right]$, while, the matrix coefficients are described as follows

$$
\begin{aligned}
& \hat{A}=\left[\begin{array}{cc}
A_{a} & 0 \\
-\gamma_{d} C_{a} & \psi_{d}
\end{array}\right], \widehat{B}=\left[\begin{array}{c}
B_{a} \\
0
\end{array}\right], \widehat{E_{1}}=\left[\begin{array}{c}
E_{a} \\
0
\end{array}\right], \\
& \widehat{E_{2}}=\left[\begin{array}{c}
0 \\
\gamma_{d}
\end{array}\right], \text { and } C_{a}=\left[\begin{array}{ll}
C & 0
\end{array}\right] .
\end{aligned}
$$

Finally, the control signal is define by:

$$
u_{a}(k)=-\boldsymbol{K}^{T} \hat{x}(k)=-\left[\begin{array}{ll}
\boldsymbol{K}_{1} & \boldsymbol{K}_{2}
\end{array}\right]\left[\begin{array}{c}
x_{a}(k) \\
\rho(k)
\end{array}\right]
$$

The choice of the vector gain $\boldsymbol{K}$ must ensure the stability of the closed loop system while maintaining an asymptotic follow-up of the reference. This vector results from the minimization of the following quadratic criterion:

$$
J\left(u_{a}(k)\right)=\sum_{k=1}^{\infty} \hat{x}(k)^{T} Q \hat{x}(k)+u_{a}(k)^{T} R u_{a}(k)
$$

The matrix $Q$ is semi definite positive, while the coefficient $R>0$.

\section{Experimental results}

As illustrated by Fig.5, a bench of experimental test was built to validate the strategy suggested for control in tension of an autonomous unit of generation distributed intended to feed from the electric charges of various natures. The bench of test consists of a rectifier intended to operate in source of continuous tension, an inverter SEMIKRON with filter LC of output, as well as panoply of electric charges. The two loops associated with both control current and tension is implemented in a platform dSPACE MicroAutoBox and requires only the measurement of the both output current and voltage. The execution time of the whole of the controller is about $20.1 \mu \mathrm{s}$, with the result that the strategy suggested does not require an excessive computing time.

The effectiveness of the strategy of control proposed in the continuation of the trajectories and the rejection of disturbance is shown through Fig. 6, and Fig.7. Those figures respectively characterize the response of the system for the linear load, the nonlinear load, and the level of load. The results obtained corroborate perfectly with those anticipated. The linear load is represented by a resistive load of $18 \Omega$, while the nonlinear load is consisted a rectifier with diodes coupled with a circuit RLC $(R=123 \Omega, L=500 \mu H, C=1 \mathrm{mF})$. The measurement of the harmonic rate of distortion of the output voltage is equal to $0.8 \%$ for the linear load, and to
$2.7 \%$ for the nonlinear load, which enables us to conclude the effectiveness of the control strategy proposed.

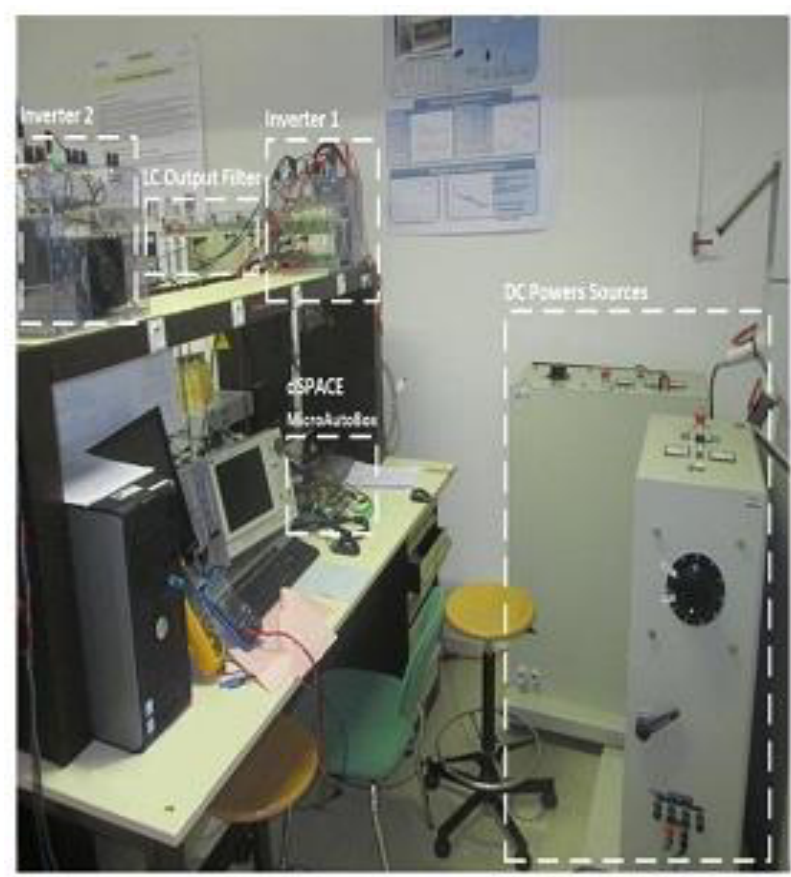

Fig. 5. Bench of experimental test.

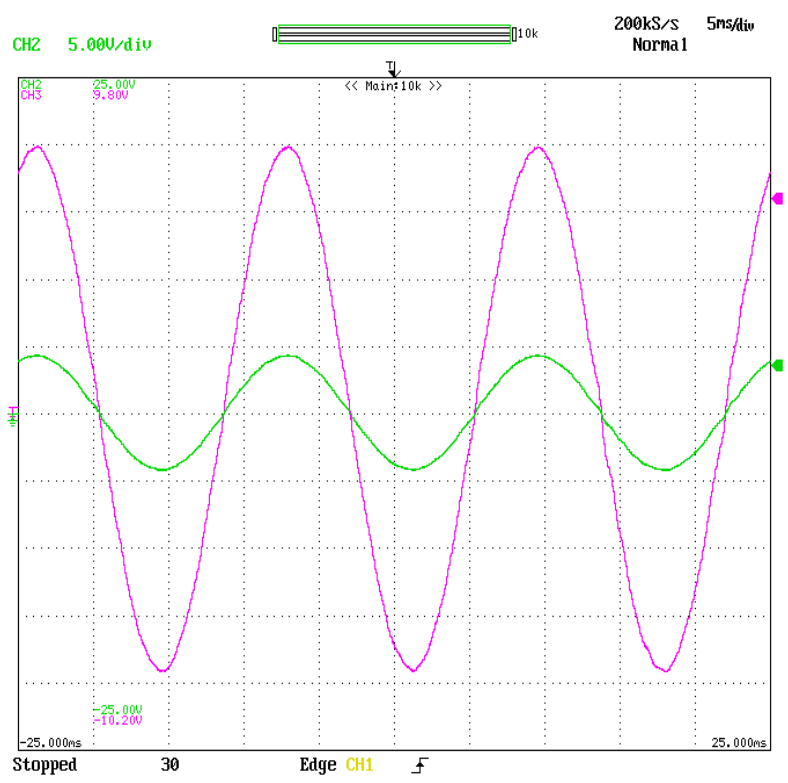

Fig. 6. Experimental results: Response of the system to a linear load, voltage curve [Pink (axis-X: 5ms/div; axis-Y: $40 \mathrm{~V} / \mathrm{div}$ )] and of the charging current [Green: (axis-X: 5ms/div; axis-Y: 10A/div)]. 


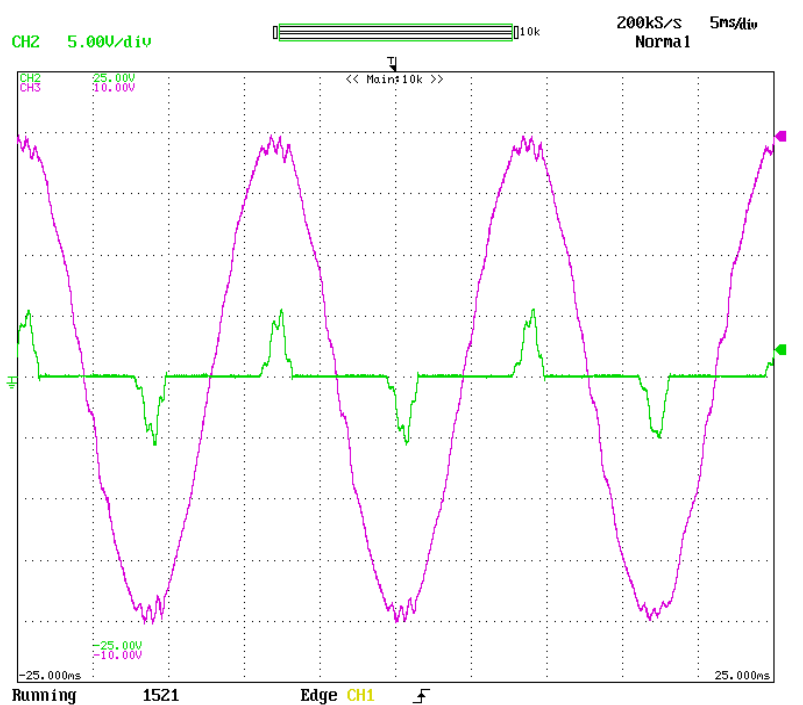

Fig. 7. Experimental results: Response of the system to a nonlinear load, voltage curve [Pink (axis-X: $5 \mathrm{~ms} / \mathrm{div}$; 40V/div)] and of the load current [ Green: (axis-X: $5 \mathrm{~ms} / \mathrm{div} ; 10 \mathrm{~A} / \mathrm{div})]$.

\section{Conclusion}

In this paper, we had to deal with the problematic appropriate to the voltage control of an autonomous distributed generator using a control strategy in nested loops has been proposed. This strategy aims to perform the synthesis in an optimal way, based on a multiresonant control with stabilizing feedback state. Several experimental results are obtained to confirm and validate the proposed control strategy. In perspective, we plan to use the same concept of resonant control while developing an adaptive control structure in frequency and gain for a micro-array application.

\section{References}

1. R. Ortega, E. Figueres, G. Garcerá, C. Trujillo, and D. Velasco, "Control techniques for reduction of the total harmonic distortion in voltage applied to a single-phase inverter with nonlinear loads: Review," Renewable and Sustainable Energy Reviews, vol. 16, pp. 1754-1761, 2012.

2. M. R. Miveh, M. F. Rahmat, A. A. Ghadimi, and M. W. Mustafa, "Control techniques for three-phase four-leg voltage source inverters in autonomous microgrids: A review," Renewable and Sustainable Energy Reviews, vol. 54, pp. 1592-1610, 2016.

3. A. Hasanzadeh and H. Mokhtari, "A simplified droop method implementation in parallel UPS inverters with proportional-resonant controller," Iranian Journal of Science and Technology, vol. 33, p. 163, 2009.

4. A. R. Monter, E. J. Bueno, A. García-Cerrada, F. J. Rodríguez, and F. M. Sánchez, "Detailed analysis of the implementation of frequency-adaptive resonant and repetitive current controllers for grid-connected converters," Electric Power Systems Research, vol. 116, pp. 231-242, 2014.

5. N.F Guerrero-Rodriguez and A.B Rey-Boue, "Adaptivefrequency Resonant Harmonic-Compensator structure for a 3-phase grid-connected photovoltaic system," Energy Conversion and Management, vol. 87, pp. 328-337, 2014.

6. J. M. Guerrero, L. Hang, and J. Uceda, "Control of distributed uninterruptible power supply systems," IEEE Transactions on Industrial Electronics, vol. 55, pp. 28452859, 2008.

7. R. S. Rao, K. Ravindra, K. Satish, and S. Narasimham, "Power loss minimization in distribution system using network reconfiguration in the presence of distributed generation," IEEE transactions on power systems, vol. 28, pp. 317-325, 2013.

8. A. Saim, R. Mellah, A. Houari, M. Machmoum, and A. Djerioui, "Adaptive resonant based multi-loop control strategy for parallel distributed generation units in standalone microgrid application," Electric Power Systems Research, vol. 143, pp. 262-271, 2017.

9. P. Sreekumar and V. Khadkikar, "A New Virtual Harmonic Impedance Scheme for Harmonic Power Sharing in an Islanded Microgrid," IEEE Trans. Power Deliv., vol. 31, no. 3, pp. 936-945, Jun. 2016.

10. A. D. Paquette and D. M. Divan, "Providing Improved Power Quality in Microgrids: Difficulties in Competing with Existing Power-Quality Solutions," IEEE Ind. Appl. Mag., vol. 20, no. 5, pp. 34-43, Sep. 2014.

11. M. Aamir, K. A. Kalwar, and S. Mekhilef, "Review: Uninterruptible Power Supply (UPS) system," Renewable and Sustainable Energy Reviews, vol. 58, pp. 1395-1410, 2016.

12. M. Kale, M. Karabacak, W. Kruschel, F. Kilic, and P. Zacharias, "Chattering free robust control of LCL filter based shunt active power filter using adaptive second order sliding mode and resonant controllers," International Journal of Electrical Power \& Energy Systems, vol. 76, pp. 174-184, 2016.

13. J. Rodriguez, J. Pontt, C. A. Silva, P. Correa, P. Lezana, P. Cortés, and U. Ammann, "Predictive current control of a voltage source inverter," IEEE Transactions on Industrial Electronics, vol. 54, pp. 495-503, 2007.

14. M. Rivera, V. Yaramasu, A. Llor, J. Rodriguez, B. Wu, and M. Fadel, "Digital predictive current control of a threephase four-leg inverter," IEEE Transactions on Industrial Electronics, vol. 60, pp. 4903-4912, 2013.

15. P. Cortes, J. Rodriguez, C. Silva, and A. Flores, "Delay compensation in model predictive current control of a three-phase inverter," IEEE Transactions on Industrial Electronics, vol. 59, pp. 1323-1325, 2012.

16. M. Pichan, H. Rastegar, and M. Monfared, "Deadbeat control of the stand-alone four-leg inverter considering the effect of the neutral line inductor," IEEE Transactions on Industrial Electronics, 2016. 